10,19

\title{
Уравнение состояния и статистическое распределение в макроскопической системе
}

\author{
(C) Н.Н. Горобей, А.С. Лукьяненко \\ Санкт-Петербургский политехнический университет Петра Великого, \\ Санкт-Петербург, Россия \\ E-mail: n.gorobey@mail.ru
}

Поступила в Редакцию 24 августа 2020 г.

В окончательной редакции 24 августа 2020 г.

Принята к публикации 25 августа 2020 г.

\begin{abstract}
Предложено представление статистической суммы для макроскопического тела в виде евклидова функционального интеграла, в котором его деформация является классическим параметром, свободным от флуктуаций. Уравнение состояния тела, связывающее его деформацию и температуру с внешней механической нагрузкой, содержится в этом представлении в виде ограничения меры интегрирования соответствующим классическим уравнением движения.
\end{abstract}

Ключевые слова: макроскопическая система, температура, деформация, квантовая механика, функциональный интеграл, статистическая сумма.

DOI: 10.21883/FTT.2020.12.50295.175

\section{1. Введение}

В настоящей работе в качестве макроскопической системы будем рассматривать изолированное твердое тело, хотя выводы имеют общий характер. Термомеханические явления в изолированном твердом теле вызывают интерес, поскольку ставят вопросы, касающиеся определения таких основных макроскопических понятий, как температура и деформация. Наглядным примером этому служит известный давно термоупругий эффект $[1,2]$, в котором для большинства тел наблюдается небольшое уменьшение температуры при их растяжении (наоборот, повышение температуры при сжатии). Необычность здесь в том, что температура ансамбля гармонических осцилляторов (в рамках модели Эйнштейна) пропорциональна полной энергии колебаний, а при любой деформации твердого тела его энергия возрастает. Эффект объясняется ангармонизмом межатомного взаимодействия. Другой эффект, связанный с ангармонизмом, - термическое расширение [3]. Оба эти эффекта имеют простое и наглядное микроскопическое объяснение. В частности, термоупругий эффект является следствием уменьшения энергии колебаний ангармонического осциллятора при его растяжении [4] как следствие теоремы об адиабатических инвариантах [5]. Термическое расширение объясняется увеличением среднего смещения атома при увеличении энергии его колебаний в асимметричной потенциальной яме [3]. Связанная с упомянутыми эффектами макроскопическая деформация тела, находится просто суммированием всех средних смещений атомов. Однако, возможен и другой взгляд на эту деформацию [6]. Поскольку речь идет о макроскопической величине, мы можем пренебречь флуктуациями смещений отдельных слагаемых большой суммы и рассматривать ее изменения независимо от статистики (классической или квантовой). В таком случае, наоборот, закономерности макроскопической деформации тела будут накладывать ограничения на статистическое распределение, модифицируя его. Возможность модификации статистического распределения следует хотя бы из того, что распределение Гиббса предсказывает флуктуации энергии системы, тогда как для изолированного тела его энергия является константой [7]. В настоящей работе предложена модификация статистической суммы изолированного тела, которая получается путем перехода к мнимому времени в квантовой механике с дополнительными условиями, не нарушающими унитарность (сохранение вероятности) в теории.

В следующем разделе определяется модифицированный оператор эволюции в квантовой теории, который представлен функциональным интегралом Фейнмана [8]. Затем, переходя к мнимому времени виковским поворотом, тем же функциональным интегралом $[8,9]$ представляем модифицированную статистическую сумму, в которой уравнение для макроскопической деформации играет роль уравнением состояния тела.

\section{2. Квантовая теория с классическими степенями свободы}

Унитарный оператор эволюции

$$
\hat{U}=\exp \left(-\frac{i}{\hbar} \hat{H} t\right),
$$

где $\hat{H}$ - оператор Гамильтона системы, может быть представлен своим ядром в виде функционального ин- 
теграла Фейнмана

$$
K\left(q, 0 ; q^{\prime}, t\right)=\int \prod_{t} d q(t) \exp \left[\frac{i}{\hbar} \int_{0}^{t} d t L(q, \dot{q}],\right.
$$

где $L(q, \dot{q})$ - функция Лагранжа классической системы. Для большой системы следует ожидать, что поведение некоторых степеней свободы в квантовой теории не будет отличаться от классической. Например, в случае атомной цепочки (модель макромолекулы) так ведет себя ее длина, равная сумме всех межатомных расстояний. На основании закона больших чисел флуктуации много меньше самой этой величины, и мы на практике всегда ими пренебрегаем. Остается только формализовать эту констатацию в квантовой теории, не нарушая при этом унитарности.

Пусть $\xi$ - та самая динамическая переменная, от которой мы ожидаем классическое поведение в квантовой теории. Легко увидеть, что существует линейное ортогональное преобразование координат, в результате которого кинетическая энергия, отвечающая этой переменной, выделяется отдельным слагаемым

$$
L=\frac{1}{2} m_{\xi} \dot{\xi}^{2}+K(\dot{u})-V(\xi, u)
$$

где $u$ - дополнительные обобщенные координаты. Наша цель будет достигнута, если мы добавим уравнение движения

$$
m_{\xi} \ddot{\xi}+\frac{\partial V}{\partial \xi}=0
$$

к исходной функции Лагранжа в качестве дополнительного условия с соответствующим множителем Лагранжа $\lambda$ :

$$
\tilde{L}=L-m_{\xi} \dot{\lambda} \dot{\xi}+\lambda \frac{\partial V}{\partial \xi} .
$$

При этом мы отбрасываем полную производную. Очевидно, такая модификация не меняет динамическое содержание теории на классическом уровне, но соответствующая часть функции Гамильтона существенно меняет форму

$$
\tilde{H}=\frac{1}{m_{\xi}}\left(p_{\lambda} p_{\xi}-\frac{1}{2} p_{\lambda}^{2}\right)+h\left(\xi, u, p_{u}\right) .
$$

Как следствие, в квантовой теории модифицированный оператор эволюции имеет следующее представление в виде функционального интеграла

$$
\begin{aligned}
\tilde{K}\left(\xi, u, 0 ; \xi^{\prime}, u^{\prime}, t\right)= & \int \prod d \xi d u \delta\left(m_{\xi} \ddot{\xi}+\frac{\partial V}{\partial \xi}-F\right) \\
& \times \exp \left[\frac{i}{\hbar} \int d t L(\xi, u, \dot{\xi}, \dot{u})\right] .
\end{aligned}
$$

Интеграл (7) на конфигурационном пространстве получается из функционального интеграла на модифицированном фазовом пространстве с функцией Гамильтона (6), который следует брать в качестве исходного [10]. Видно, что суммирование теперь ограничено траекториями $\xi(t)$, которые подчиняются классическому уравнению движения (4) с заданными начальной $\xi$ и конечной $\xi^{\prime}$ точками. При этом волновая функция системы получает дополнительную зависимость от скорости $\dot{\xi}$ : $\psi(\xi, \dot{\xi}, u)$.

\section{3. Модифицированная статистическая сумма}

Переход от квантовой механики к классической статистической механике осуществляется виковским поворотом в комплексной плоскости времени [9]. Более точно эта процедура сводится к замене

$$
t \rightarrow \tau=-i h \beta \sigma, \quad \beta=\frac{1}{k T}
$$

и взятию следа от унитарного оператора эволюции (1). Далее мы полагаем всюду для простоты $\hbar=1$. В результате получаем статистическую сумму рассматриваемой системы в виде функционального интеграла Фейнмана в евклидовой форме

$$
Z=\int_{\text {periodic }} \prod_{q(\tau)} d q(\tau) \exp \left[-\int_{0}^{\beta} d \tau L(q, \dot{q})\right]
$$

Суммирование здесь ведется по всем периодическим на промежутке $0 \leq \tau<\beta$ траекториям системы, точкой теперь обозначена производная по $\tau$.

Теперь проделаем эту процедуру для модифицированного оператора эволюции в представлении (7). В результате приходим к модифицированной статистической сумме в виде функционального интеграла Фейнмана в евклидовой форме

$$
\begin{aligned}
& \tilde{Z}=\int_{\text {periodic }} \prod_{u(\tau)} d \xi(\tau) d u(\tau) \\
& \times \delta\left(-m_{\xi} \ddot{\xi}+\frac{\partial V}{\partial \xi}-F\right) \exp \left[-\int_{0}^{\beta} d \tau L(\xi, u, \dot{\xi}, \dot{u})\right] .
\end{aligned}
$$

Суммирование здесь ведется по всем периодическим траекториям дополнительных переменных $u(\tau)$ и гладкой замкнутой $\left(\xi=\xi^{\prime}, \dot{\xi}=\dot{\xi}^{\prime}\right)$ траектории макроскопической деформации $\xi(\tau)$. В это уравнение движения мы добавили внешнюю силу $F$. Соответствующую ей потенциальную энергию взаимодействия в действие не добавляем, так как далее будет вычисляться энергия рассматриваемого тела, к которой эта величина не относится. Заметим, что по совпадающим граничным точкам (граничной точке $\xi$ ) траектории деформации в мнимом времени суммирование не проводится. Таким образом, модифицированная статистическая сумма зависит от двух переменных $\xi, T$, которые являются макроскопическими параметрами состояния. Макроскопическая деформация определяется „уравнением движения“ 
в мнимом времени

$$
-m_{\xi} \ddot{\xi}+\frac{\partial V}{\partial \xi}-F=0
$$

Обратим внимание, что „ускорение“ в этом уравнении имеет противоположный знак по сравнению с движением в реальном времени. Это эквивалентно изменению знака потенциального рельефа, т.е. теперь мы изучаем движение вблизи максимума потенциальной энергии. Чтобы траектория вернулась в исходную точку, она всегда должна находиться на самом максимуме. Таким образом, макроскопическая деформация определяется из условия равновесия

$$
\left\langle\frac{\partial V}{\partial \xi}\right\rangle-F=0,
$$

в котором усреднение означает функциональное интегрирование по дополнительным переменным в (10). Это и есть уравнение состояния тела, которое определяет зависимость макроскопической деформации от внешней силы и температуры. При этом температура тела определяется его внутренней энергией, которую определяем соотношением

$$
W=\frac{\partial \ln \tilde{Z}}{\partial \beta} .
$$

\section{4. Заключение}

Таким образом, термомеханические явления в макроскопическом теле не являются однозначно прямым следствием (суммой) элементарных микроскопических эффектов. В частности, деформация макроскопического тела следует законам классической механики с добавлением к ним закона больших чисел, а статистическое усреднение, которое также требуется в формулировке этих макроскопических закономерностей, само формулируется в заданных макроскопических ,рамках“. Иными словами, закономерности разных уровней в большой системе могут быть сформулированы самосогласованным образом. Этот подход не отменяет традиционное „суммирование“ элементарных процессов. Однако, результат „суммирования“ может быть найден независимо. В любом случае поведение системы на всех уровнях определяется ее структурой (гамильтонианом) и заданными внешними условиями.

\section{Список литературы}

[1] J.P. Joule. Proc. R. Soc. 8, 564 (1857).

[2] W. Thompson (Lord Kelvin). Trans. Roy. Soc. Edinburgh 20, 261 (1853).

[3] Ч. Киттель. Введение в физику твердого тела. Гостехиздат, M. (1957). 524 c.

[4] A.I. Slutsker, V.P. Volodin. Thermochim. Acta 247, 111 (1994).
[5] Л.Д. Ландау, Е.М. Лифшиц. Механика. Изд-во физ.-мат. лит., М. (1958). 206 с.

[6] Н.Н. Горобей, А.С. Лукьяненко. ФТТ 56, 2187 (2014).

[7] Л.Д. Ландау, Е.М. Лифшиц. Статистическая физика. Наука, М. (1976). Ч. 1. 583 c.

[8] Р. Фейнман, Хиббс. Квантовая механика и интегралы по траекториям. Мир, М. (1968). 383 с.

[9] Р. Фейнман. Статистическая механика. Мир, М. (1975). $407 \mathrm{c}$.

[10] А.А. Славнов, Л.Д. Фаддеев. Введение в теорию калибровочных полей. Мир, М. (1988). 272 с.

Редактор Т.Н. Василевская 